\title{
The GiSAS study: Rationale and design of a pragmatic randomized controlled trial on aripiprazole, olanzapine and haloperidol in the long-term treatment of schizophrenia
}

\author{
Alberto Parabiaghi a,*, Barbara D'Avanzo a, Mauro Tettamanti ${ }^{\mathrm{b}}$, Angelo Barbato ${ }^{\mathrm{a}}$ \\ and GiSAS Study Group ${ }^{1,2}$
}

a Epidemiology and Social Psychiatry Unit, 'Mario Negri' Institute for Pharmacological Research, Via La Masa 19, 20156 Milan, Italy

b Laboratory of Geriatric Neuropsychiatry, 'Mario Negri' Institute for Pharmacological Research, Via La Masa 19, 20156 Milan, Italy

\section{A R T I C L E I N F O}

\section{Article history:}

Received 25 November 2010

Received in revised form 29 March 2011

Accepted 21 April 2011

Available online 30 April 2011

\section{Keywords:}

Antipsychotic

Schizophrenia

Pragmatic trial

Metabolic syndrome

Drug discontinuation

\begin{abstract}
A B S T R A C T
Given the controversy about the comparative efficacy of first- compared with second-generation antipsychotics in the treatment of schizophrenia, more large-scale evidence is needed to guide clinicians in their prescriptions. Most randomized controlled trials (RCTs) were conducted in centers of excellence on highly selected samples, poorly representative of real-world patients, and often suffered conflicts of interest as they were sponsored by drug companies.

The primary aim of the present study is to compare the effectiveness of haloperidol, olanzapine and aripiprazole in a representative sample of schizophrenia patients. The GiSAS trial is an open-label, independent, pragmatic RCT in Italian community-based public psychiatric services. At least 260 patients meeting the DSM-IV criteria for schizophrenia will be randomly allocated to one of the study drugs and followed up for one year. A two-year observational phase will follow. The primary outcome for tolerability will be the onset of metabolic syndrome. The primary endpoint for effectiveness will be discontinuation of antipsychotic monotherapy. Secondary measures include global functioning, time to discontinuation due to side-effects, change of lipid profile, extrapyramidal symptoms and other adverse effects. In the last four years, the GiSAS study group has been working to implement this multicenter RCT. The trial mechanism is now fully functional and working. As of end of February 2011, 260 subjects were randomized by 54 study investigators in 33 out of 43 participating centers.
\end{abstract}

(c) 2011 Elsevier Inc. All rights reserved.

Abbreviations: ATP, Adult Treatment Panel III;CATIE, Clinical Antipsychotic Trials of Intervention Effectiveness;CUtLASS, Cost Utility of the Latest Antipsychotic Drugs in Schizophrenia;EPS, Extrapyramidal symptoms;EUFEST, European First Episode Schizophrenia Trial;FGAs, First-generation antipsychotics;GAF, Global Assessment of Functioning;GiSAS, Italian Group for the Study of Second-generation Antipsychotics;ITT, Intention to treat;LUNSERS, Liverpool University Neuroleptic Side Effect Rating Scale;SGAs, Second-generation antipsychotics.

it Trial Registration. ClinicalTrials.gov Identifier NCT01052389.

* Corresponding author. Tel.: + 3902 39014602; fax: + 3939014300.

E-mail addresses: alberto.parabiaghi@marionegri.it (A. Parabiaghi), barbara.davanzo@marionegri.it (B. D'Avanzo), mauro.tettamanti@marionegri.it (M. Tettamanti), angelo.barbato@marionegri.it (A. Barbato).

1 http://www.gisas.marionegri.it.

2 eXeCUTIVE CommitTeE: Angelo Barbato (Principal Investigator), Barbara D'Avanzo (Trial Coordinator), Alberto Parabiaghi (Project Director), Mauro Tettamanti (Trial Statistician). STEERING commitreE: Clive E. Adams, Eugenio Aguglia, Mariano Bassi, Giancarlo Breviario, Massimo Casacchia, Antonio Colotto, Arcadio Erlicher, Luigi Ferrannini, Farida Ferrato, Bruno Gentile, Maurizio Marcenaro, Antonino Mastroeni, Massimo C. Mauri, Mauro Percudani, Vincenzo Scalfari, Giovanni Smerieri, Marco Vaggi. STudy teAm: Grazia Buratti (Monitor), Lucia Buratti (Monitor), Andrea De Micheli, Karin Furlato, Simona D'Onofrio, Ilaria Mariannantoni, Filippo Rapisarda (Trial Manager), Francesca Riccardi, Alessandra Ruberto, Irene Ruggirello, Ilaria Santini, Federica Trivelli, Angelida Ullo. STUDY INVESTIGATORS: Germana Agnetti, Stefano Bignotti, Chiara Bufalino, Dario Cannavò, Carlo I. Cattaneo, Laura Comino, Angela D'Aloise, Ilaria F. De Gaspari, Vincenzo Florio, Antonio Francomano, Vincenzo Fricchione Parise, Liliana Gandolfo, Emanuela Ghinaglia, Lucio Ghio, Christian Groppi, Aristotele Hadjichristos, Adelia Lucattini, Samuele Manfrinati, Werner Natta, Gaetano Pinto, Rocco Pollice, Paolo Pomero, Danilo Rigamonti, Bartolomeo Senette. 


\section{Introduction}

Schizophrenia is a serious and disabling mental illness causing positive symptoms (such as delusions and hallucinations) and negative ones (such as lack of motivation and social withdrawal). Schizophrenic disorders can have farreaching negative effects on personality, cognition, global functioning and quality of life and usually need long-term therapy.

The short-term benefits of first-generation antipsychotics (FGAs) in controlling positive symptoms are well documented, as are their high rates of extrapyramidal side-effects. However, long-term data are still few and there is no convincing evidence that FGAs have any effect on the negative symptoms [1-3]. Second-generation antipsychotics (SGAs) claim to be more effective and better tolerated than FGAs. However, early reviews questioned their advantages $[4,5]$. Although there is evidence of a lower risk of neurologic side-effects, they are reported to cause metabolic side-effects like weight gain, dyslipidemia and impaired fasting glucose [6]. Only clozapine proved clearly superior and was effective in non-responders to other antipsychotics, but severe sideeffects limit its use in community settings $[7,8]$.

Initial enthusiasm for the "atypical" effect of SGAs has gradually waned, and concern has grown about their high costs $[9,10]$. Researchers have become increasingly suspicious about the evidence on antipsychotics, most of which comes from industry-sponsored trials $[11,12]$.

In a recent meta-analysis some SGAs showed more effect than FGAs in terms of symptom relief, with small effect sizes for amisulpride, olanzapine and risperidone and only a medium effect size for clozapine [13]. However, in another meta-analysis focusing on the metabolic side-effects of SGAs, olanzapine and clozapine had the worst profile [14].

Recently three landmark pragmatic and independent studies have cast further doubts on the effectiveness of SGAs compared to FGAs in schizophrenia (see Table 1) [1523]. The US CATIE phase 1 study was a double-blind trial in which 1493 patients with chronic schizophrenia were randomized to one of the SGAs olanzapine, quetiapine, risperidone or to the FGA perphenazine, and followed up for 18 months [17]. The CATIE trial included a phase 2 where discontinuers were re-randomized to an open-label comparison between clozapine and the remaining SGAs [18]. The UK CUtLASS study comprised two smaller open-label trials comparing FGAs and SGAs in terms of quality of life at one year $[19,20]$. CATIE and CUtLASS both found no significant differences between SGAs and FGAs in terms of effectiveness or tolerability $[17,19]$. However, they found clozapine more effective in refractory schizophrenia, and FGAs were more cost-effective than SGAs $[18,20]$.

The European First Episode Schizophrenia Trial (EUFEST) study compared the effectiveness of low-dose haloperidol or SGAs in first-episode schizophrenia [21]. Treatment was discontinued more by patients on haloperidol, but since discontinuation rates were not consistent with symptomatic improvement the superiority of SGAs was not confirmed. Finally, two papers presenting data from CATIE and EUFEST reported no significant differences between FGAs and SGAs in terms of cognitive test performance [22,23].

\section{Material and methods}

\subsection{Study hypothesis}

All these findings fueled disagreement among researchers and clinicians instead of resolving the controversy about the comparative effectiveness of antipsychotic medications. This may be partly due to the fact that SGAs are not a homogeneous category, as they differ in many properties and comprise both old drugs, like clozapine and amisulpride, and the newer one, like aripiprazole and ziprasidone. In a recent critical review Leucht and colleagues concluded that although atypical antipsychotics are not a breakthrough, the overall evidence in favor of some of them is consistent. Thus, the debate for or against SGAs seems to be influenced more by values than by data [24].

These conflicting views call for more pragmatic evidence to guide clinicians. Therefore, the Italian Group for the Study of Second-generation Antipsychotics (GiSAS) decided to design a pragmatic, open-label RCT to compare old and new antipsychotics in people with schizophrenia treated in routine Italian clinical settings.

Since the controversy on the comparative efficacy of SGAs over FGAs was not settled by the latest pragmatic RCTs we proposed a trial focused on tolerability. Adverse reactions like metabolic disturbances or extrapyramidal symptoms (EPS) are extremely common with antipsychotic treatment. Moreover, best available evidence shows that differences in terms of side-effects among SGAs are larger and clinically more relevant than differences in efficacy [14]. Thus, GiSAS was designed to find out whether any one of the selected

Table 1

Examples of independently funded pragmatic RCT on long-term effectiveness of antipsychotic therapy in schizophrenia.

\begin{tabular}{|c|c|c|c|c|}
\hline & CATIE - phase 1 & CUtLASS 1 & EUFEST & GiSAS \\
\hline Design & Double-blind & Open-label (rater-blinded) & Open-label & Open-label (analyst-blinded) \\
\hline Follow-up & 18 months & 12 months & 12 months & 12 months \\
\hline Study drugs & $\begin{array}{l}\text { Olanzapine vs. } \\
\text { perfenazine vs. } \\
\text { quetiapine vs. } \\
\text { risperidone }\end{array}$ & FGA vs. SGA & $\begin{array}{l}\text { Haloperidol vs. [amisulpride, } \\
\text { olanzapine, quetiapine, ziprasidone] }\end{array}$ & $\begin{array}{l}\text { Aripiprazole vs. [olanzapine, } \\
\text { haloperidol] }\end{array}$ \\
\hline Primary endpoint & $\begin{array}{l}\text { Time to study drug } \\
\text { discontinuation }\end{array}$ & Quality of life & Time to study drug discontinuation & $\begin{array}{l}\text { Staying on study drug without } \\
\text { developing metabolic syndrome }\end{array}$ \\
\hline Participating centers & 57 US & $14 \mathrm{UK}$ & 50 (14 UE countries) & 43 ITA \\
\hline Patients (no.) & 1432 & 227 & 498 & 260 \\
\hline Funding & NIMH & NHS & EGRIS (Pfizer, AstraZeneca, Sanofi-Aventis) & MNegri (Bristol-Myers Squibb) \\
\hline
\end{tabular}


antipsychotics was associated with better treatment continuation and caused less harm than the others.

The study drugs were selected taking account of tolerability and current prescribing practice in Italy. We opted for a comparison between two SGAs and one FGA. Clozapine was excluded because of its peculiar side-effect profile and difficult management. Thus, among the other SGAs (i.e. amisulpride, risperidone, olanzapine, quetiapine, and aripiprazole) we selected aripiprazole and olanzapine.

When the trial was being planned (2006) aripiprazole was the latest and promising antipsychotic with a good but only preliminary reputation for tolerability (i.e. metabolic and EPS) and a still doubtful reputation for effectiveness. It was believed to work as a D2-antagonist in pathways where an excess of dopamine produces psychosis, and as a D2-agonist at sites where low dopaminergic tone would produce motor side-effects and increased prolactin release [25]. It was associated with a low likelihood of inducing EPS, sedation, QTc prolongation, weight gain and metabolic abnormalities. However, evidence on its effectiveness and tolerability was still scant and its hypothetically different profile of therapeutic and adverse effects was not yet proven [26].

Olanzapine is a widely used SGA and has for many years been the most prescribed antipsychotic in Italy [27]. It had an excellent reputation for efficacy, and was known to cause few EPS; however, it did have adverse effects on body weight $[9,28]$.

Lastly, we selected haloperidol a highly potent and effective FGA and the most widely used comparator in clinical trials investigating the efficacy of antipsychotic drugs for schizophrenia [9]. The use of haloperidol as control drug for randomized trials of new antipsychotics has been questioned for its propensity to cause EPS [29]. Movement disorders, however, mostly arose with high doses of haloperidol $[5,29,30]$, and, in fact, Geddes and colleagues (2000) concluded that trials showing SGAs to be superior to haloperidol used too high doses of the latter [5]. It has therefore been argued that haloperidol's comparative effectiveness might improve with a more prudent dosing approach, although this has not been confirmed in recent reviews [9]. On the other hand, haloperidol has low propensity to cause metabolic side-effects, although this too has never been thoroughly investigated [31]. Haloperidol is the most prescribed FGA worldwide and is still widely prescribed in Italy, where a strong tradition of low dosing may have resulted in less criticism [27].

The GiSAS trial was conceived to be large enough to identify small to moderate differences in treatment effects and simple enough to be introduced in non-academic community mental health services. Our purpose was to compare FGAs and SGAs in terms of tolerability and effectiveness, so as to detect meaningful differences. Therefore we focused on two sound endpoints: metabolic syndrome and treatment discontinuation. As the tendency of haloperidol to cause more EPS was well known, and available findings did not suggest relevant differences in terms of EPS between olanzapine and aripiprazole, those neurological side-effects were adopted as secondary endpoints [2,4,26].

The metabolic syndrome is a constellation of closely related risk factors and represents a way of assimilating risk across the various pathogenic pathways related to obesity. In this study metabolic syndrome was diagnosed on the basis of at least three of the criteria indicated by the Adult Treatment Panel III (ATP III) [32]:

1. Abdominal obesity (waist circumference $>102 \mathrm{~cm}$ in men and $>88 \mathrm{~cm}$ in women);

2. Fasting triglycerides $\geq 150 \mathrm{mg} / \mathrm{dL}$;

3. High-density lipoprotein $<40 \mathrm{mg} / \mathrm{dL}$ in men or $<50 \mathrm{mg} / \mathrm{dL}$ in women;

4. High blood pressure $\geq 130 / 85 \mathrm{~mm} \mathrm{Hg}$ or on antihypertensive medication;

5. Fasting glucose $\geq 110 \mathrm{mg} / \mathrm{dL}$ or on insulin or hypoglycemic medication.

Patients with schizophrenia have a mortality rate two to three times higher than the general population mainly due to suicide and natural causes, including cardiovascular diseases $[33,34]$. Although their lifestyle may contribute to the higher mortality [35], a role has also been attributed to antipsychotic medications, through direct effects on cardiac repolarization, possibly leading to sudden cardiac death, and to metabolic syndrome [34,36,37].

Long-term antipsychotic prescription has become one of the cornerstones of treatment for psychotic disorders. Patients on antipsychotic medication do better overall and early cessation often results in higher relapse rates, hospitalization and risk of death and violence [38-40]. Nevertheless, medication management remains a serious problem as some reports give rates of drug non-adherence around 50\% [41]. Poor efficacy and intolerable side-effects are common and contribute to premature discontinuation. The CATIE and EUFEST trials adopted time to all-cause treatment discontinuation as primary endpoint. Though poor symptom response has been indicated as the main reason for antipsychotic discontinuation in clinical trials [42,43], antipsychotic discontinuation is considered a global indicator of treatment tenure and a proxy measure of efficacy, safety, and tolerability.

\subsection{Study aims}

Given the controversy on the comparative efficacy of FGAs over SGAs, clinicians should face substantial uncertainty in the choice of the antipsychotic likely to provide greatest clinical benefit in adult patients with no specific contraindications, who have responded inadequately to previous antipsychotic medication. The clinical question posed in the GiSAS trial therefore concerns the relative tolerability and effectiveness of aripiprazole, olanzapine and haloperidol in the medium- and long-term treatment of schizophrenia, in terms of metabolic syndrome onset and drug discontinuation.

\subsection{Study design}

The GISAS trial is an open label, one-year randomized controlled trial firmly rooted in everyday clinical practice. To enhance representativeness, the inclusion criteria are wide and recruitment takes place in a broad array of clinical settings and across the various components of service provision. The sample is meant to be heterogeneous and to reflect the real population attending Italian community psychiatric services, recruiting a broad range of "real-world" patients, including those with comorbid conditions. 
Eligible subjects are randomly assigned to non-blind oral monotherapy with one of the study drugs. The study is not designed to replace any aspect of the usual clinical care. During follow-up, participants will be seen as often as is usually clinically indicated. Each patient will be examined in the respective recruiting center by the usual clinician.

At the end of the 12-month follow-up, all those randomized will enter a two-year prospective observational study.

\subsection{Sample selection}

Inclusion criteria:

- age 18 years and over;

- DSM-IV diagnosis of schizophrenia, based on the Mini International Neuropsychiatric Interview [44];

- patients entering the study should, according to their own judgment and in consultation with their doctor, have a condition appropriate for changing current antipsychotic treatment.

\section{Exclusion criteria:}

- metabolic syndrome diagnosed on the basis of at least three of the criteria listed above:

- diabetes mellitus type II;

- any organic condition clearly contraindicating treatment with any of the drugs (e.g. pregnancy or breast-feeding);

- known ineffectiveness or intolerance of one of the study drugs, which is consequently contraindicated;

- patients who have never been exposed to antipsychotic drugs;

- in the clinician's opinion, it is unlikely that the patient can be followed-up for the whole study (one year).

\subsection{Recruitment}

Recruitment is conducted in a broad array of community settings: outpatient clinics, acute hospital units, residential facilities and day centers. Patients meeting the inclusion criteria are asked to participate and, after giving fully informed consent, can access randomization. Once randomized, they are included in the study if they take at least one dose of the assigned medication. The recruitment forms of patients excluded are filed and periodically reconsidered for inclusion.

\subsection{Randomization}

Eligible patients are randomized to the three treatment arms following a 1:1:1 procedure. Randomization is stratified by site. Although the number of criteria already satisfied in a patient at study entry may be a powerful predictor of developing metabolic syndrome during follow-up, the small numbers recruited per center make it impossible to stratify for this variable too. A secondary statistical analysis of the primary outcome will adjust for the number of criteria already met at study entry.

The allocation sequence (computer-generated random sequence) has been registered before the trial's start. Investigators are unaware of how exactly the chosen randomization method is being implemented.

Central randomization by telephone with an interactive voice response system has been adopted. Investigators assess eligibility, gain consent, and make the decision to enroll a patient at the participating site, then call the randomization service to get the treatment allocation and the patient's code. All records on the randomization database (e.g. failed calls, unregistered randomizations) will be checked to detect any attempts to decipher allocation.

\subsection{Outcomes}

The onset of metabolic syndrome was adopted as primary negative endpoint. However, the trial takes account of two primary endpoints: one for tolerability and one for effectiveness $[45,46]$.

\subsubsection{Primary endpoint for tolerability}

Patients who did not develop metabolic syndrome after a year-long trial of the antipsychotic will be considered treatment successes. The onsets of metabolic syndrome, defined by meeting at least three of the above listed criteria, will be considered treatment failures even if the patients had one or two clinical signs of metabolic syndrome at study entry. The onset of metabolic syndrome must be recorded at the end of the trial or when the assigned monotherapy is stopped/changed or a second antipsychotic is added. Analyses are centralized and metabolic syndrome parameters are assessed in the same reference laboratory.

\subsubsection{Primary endpoint for effectiveness}

All-cause discontinuation of the allocated monotherapy during follow-up will be considered treatment failure. Switching to another antipsychotic, adding a second one or stopping the assigned drug is classified as study drug discontinuation. Reasons for discontinuing the assigned antipsychotic are registered but not considered in the primary outcome (Fig. 1).

Patients who meet the criteria for drug discontinuation are counted as treatment failures with regard to effectiveness but are followed up for the rest of the year.

In order to capture whether, when and why participants stop the assigned treatment or add concomitant medication, ongoing treatments are closely monitored. Occasional parenteral antipsychotic drug administration (e.g. during emergency admission) will be allowed. A temporary stop of the assigned antipsychotic (no longer than two weeks in six months) will not be considered discontinuation.

The proportions of subjects in the three study groups who discontinue treatment during the 12-month follow-up will be compared. Time to discontinuation will be taken into account in the secondary analysis.

\subsubsection{Secondary endpoints}

The following secondary endpoints will be taken into account:

- reasons for study drug discontinuation;

- Global Assessment of Functioning score (GAF) [47];

- patients' subjective reports of adverse effects, assessed by the Liverpool University Neuroleptic Side Effect Rating Scale (LUNSERS) [48];

- worsening of metabolic profile, defined as the onset of at least one metabolic syndrome criterion; 
DRUG DISCONTINUATION MODULE

\section{PLEASE MARK THE MAIN REASON FOR DRUG DISCONTINUATION}

\section{DISCONTINUATION date:}

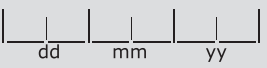
0 LACK OF EFFICACY
$\rightarrow$
(1) clinician's decision
(1) patient's decision
(2) shared decision

Please specify:

1

POOR TOLERABILITY

$\rightarrow$

(0) clinician's decision

(1) patient's decision

(2) shared decision

Please specify:

2 PATIENT'S OWN INITIATIVE

Please specify:

3

CLINICAL REMISSION

$\rightarrow$

(0) clinician's decision

(1) patient's decision

(2) shared decision

Please specify:

Fig. 1. The study drug discontinuation module in the trial monitoring form.

- onset of serum lipid abnormalities (dyslipidemia);

- waist-to-hip-ratio (ratio of waist to hip circumferences);

- electrocardiographic abnormalities;

- hyperprolactinemia;

- EPS: evaluated through physical examination by clinicians, and derived directly from specific LUNSERS items and indirectly from the use of antiparkinson drugs;

- concurrent psychotropic medication, to pragmatically evaluate the occurrence of further psychiatric symptoms or changes in different dimensions of psychopathology.

\subsection{Study medications}

All the study drugs are currently licensed and marketed in Italy for the treatment of schizophrenia. After randomization, the daily oral drug doses will be prescribed according to usual care practice and adjusted according to individual response and side-effects. Although clinicians will not be obliged to follow strict dose ranges, suggested doses are as follows:

- Aripiprazole $10 \mathrm{mg} /$ day starting dose and 10-30 mg/day dose range.

- Olanzapine $5 \mathrm{mg} /$ day starting dose and 10-20 mg/day dose range.

- Haloperidol 1-3 mg/day starting dose and 3-10 mg/day dose range.
For patients already taking an antipsychotic medication prior to study entry, tapering the previous medication over a period of at least four weeks is suggested. Reasons for being switched will be recorded and used in the secondary analyses to control for a prior disposition to resistance or intolerance. Guidelines will be provided to help physicians choose the switching strategy that is the best for each patient, with which the patient is most comfortable. The continued use of concomitant antipsychotic medication at the end of the switch period is considered a discontinuation of the allocated trial drug.

After inclusion few limits are imposed and clinicians will be free to treat patients at their own discretion. Concomitant psychotropic medication (e.g. benzodiazepines, antidepressants) or non-psychotropic drugs (e.g. anticholinergic drugs, beta-blockers, statins) are allowed and must be routinely recorded. Data on their prescription will be used in the secondary analyses.

Mood stabilizers will be allowed only for patients who were taking these medications prior to study entry, as their use is associated with metabolic disturbances.

\subsection{Masking}

Those who are directly involved in patients' care are not blinded. In blinded trials clinicians are not perfectly able to tune and adjust the optimal dose to the individual patients. 
The open-label nature of this trial enhances its feasibility, reflects real clinical practice, increases its external validity and consequently the generalizability of the results.

All the people involved in GiSAS trial coordination, supervision, and data analysis are blinded.

\subsection{Follow-up evaluations}

Patients will be assessed (a) at baseline, (b) when monotherapy is stopped or changed and (c) at 12 months. Thus, at the end of the trial all subjects are re-assessed even if they have stopped or changed the assigned drug.

During the one-year follow-up, participants will be seen as often as usually clinically indicated (about once a month). On these occasions investigators will be asked to complete a specific monitoring form to check if any change in the patient's medication has occurred (see Fig. 1).

With the exception of the centralized analyses, which are done at the Mario Negri Institute to detect metabolic disturbances, each patient is examined by the usual clinician in the respective recruiting center. Any psychosocial intervention provided by services during the study is monitored and recorded.

\subsection{Data analysis}

All analyses will be by full intention-to-treat (ITT) including all randomized participants who receive at least one dose of the investigational drugs. Patients already taking one of the study drugs at study entry can never be excluded from the ITT analysis. Data will be analyzed using a lastobservation-carried-forward approach. Patients with no follow-up data will be allocated to the outcome category of treatment failure. In addition a "per protocol" analysis will be done comparing patients who actually used the study drugs.

\subsubsection{Primary outcome indicators}

The proportion of participants in each treatment arm who do not develop metabolic syndrome during follow-up is adopted as primary endpoint for tolerability. The proportion of patients who continue the allocated antipsychotic as monotherapy in each treatment arm for the whole year is adopted as primary endpoint for effectiveness.

\subsubsection{Secondary outcome indicators}

Mean GAF scores in each treatment arm will be compared. Covariance analysis and effect size calculation will be done. Survival analysis of time to discontinuation due to treatmentrelated side effects will also be done. Subgroup comparisons will be made to analyze patients who will stop treatment due to (a) EPS and (b) onset of metabolic syndrome. The proportion of patients whose metabolic profile worsens during follow-up will be taken into account.

Mean waist-to-hip ratios in each treatment arm will be compared, covariance analysis and effect size calculation will be done. Mean LUNSERS scores in each treatment arm will be compared, covariance analysis and effect size calculation will be done. The proportion of patients developing electrocardiographic abnormalities in each treatment arm will be compared. The proportion of patients in each treatment arm who take antiparkinson drugs or extra psychotropic medica- tion will be used to pragmatically evaluate the onset of EPS or further psychiatric symptoms.

\subsection{Statistical considerations}

The main goal of the trial is to test the null hypothesis that the proportion of cases developing metabolic syndrome is similar for all three drugs. The criterion for significance (alpha) has been set at 0.05 (2-tailed). Treatments will be compared using a logistic regression model taking account of the stratification criterion used (trial site).

For all statistical analyses, sites with a small number of randomized patients will be pooled. The analyses will be conducted on the ITT population: all those who take at least one dose of study medication will be included.

If the null hypothesis of the three treatments being equal is rejected, subsequent analyses will consider each pair of drugs. Pairwise comparisons between the three treatments will ensure that the overall Type I error rate is maintained at 0.05 using a Hochberg adjustment for multiple comparisons. All three comparisons will be significant if the largest p-value is $\leq 0.05$, the two strongest comparisons will be significant if the second-smallest p-value is $\leq 0.025$, and only the strongest comparison will be significant if the smallest p-value is $\leq 0.0167$.

As secondary steps, survival analyses using Cox proportional hazards regression models and logistic regressions/ analyses of covariance will be done on the secondary outcome measures. In survival analyses, subjects still on treatment one year after randomization will be censored.

\subsubsection{Power calculation}

The criteria for significance have been set at 0.05 (twotailed), corrected for multiple testing, wherever necessary. On the basis of data extrapolated from recent studies [17,49$52]$, it is hypothesized that the percentages of patients who will develop metabolic syndrome within 12 months will be $25 \%$ in the group treated with olanzapine, $15 \%$ in the haloperidol group and $5 \%$ in the aripiprazole group.

The first step of the primary analysis is an overall 2 degrees of freedom test (using a 2 df Chi square as an approximation for the logistic regression). The proposed total sample size of 750 subjects (see below for discussion of the number) will have a power exceeding 99\% to yield a statistically significant result. If a significant difference is found, pairwise comparisons between the study drugs will be done.

Assuming that (a) the smallest detectable difference is found in the olanzapine vs. haloperidol comparison (at $\mathrm{p}=0.05$ ), (b) the second-smallest difference is found in the haloperidol vs. aripiprazole $(p=0.025)$, and $(c)$ the largest difference is between aripiprazole and olanzapine comparison $(\mathrm{p}=0.016), 250$ subjects per group will correspond to a power of, respectively, $80 \%, 93 \%$ and more than $99 \%$. If $7 \%$ of patients on aripiprazole develop metabolic syndrome, the power to detect a difference against $15 \%$ on haloperidol will be $73 \%$.

To protect against a possible drop-out rate of $5-10 \%$, about 800 patients are required for the study.

On the basis of data extrapolated from the most recent comparable study [16], it is hypothesized that retention at 
12 months will be $45 \%$ in the group treated with olanzapine, and $30 \%$ in the haloperidol group. We assume a retention rate of $60 \%$ in the group treated with aripiprazole. Using the sample size calculated above $(n=800)$, the measure of effectiveness adopted as secondary outcome (retention rate at one year) has a power of $>85 \%$ to detect significant differences (alpha $=0.05 / 0.025 / 0.016$, two-tailed) between the three pairwise comparisons, using a logistic regression.

As is common in trials, inclusion of enough patients has been a problem. Thus, having realized that the original sample size could not be achieved we had to reduce the sample to about one third of the original size. Adjustment for multiple comparisons lowers statistical power. Therefore, to preserve the study power we cut one of the planned comparisons and focused on aripiprazole vs. olanzapine, and vs. haloperidol. The less original comparison between olanzapine and haloperidol was moved to the secondary analyses.

We expected to recruit about 260 patients. Assuming that drop-out rate will be marginal (less than 10\%) data from 240 subjects should be available for analyses.

The first step of the primary analysis is still an overall 2 degrees of freedom test. The proposed sample of 240 subjects will have a power of $89 \%$ to yield a statistically significant result. If a significant difference is found, pairwise comparisons between the aripiprazole and the two other compounds will be done. Assuming that (a) the smallest detectable difference is found in the aripiprazole (5\%) vs. haloperidol (20\%) comparison (at $\mathrm{p}=0.05$ ), and $(\mathrm{b})$ the largest difference between aripiprazole (5\%) and olanzapine (25\%) (at $\mathrm{p}=0.025), 80$ subjects per group will correspond to a power of, respectively, $77 \%$ and $87 \%$.

\section{Results}

\subsection{Trial registration and conduct}

The study protocol, the study manual, the information brochure and the informed consent form have been approved by the ethics research review board of the clinical coordinating center, the Local Health Agency of Genoa ( $n^{\circ} 49549$, March 2007). The trial has been approved as an independent study aimed at improving clinical practice in health care, according to the definition of the Italian Ministerial Act 17 December 2004, and has been registered in the National Monitoring Centre for Clinical Trials and the European Clinical Trial Database (EudraCT number 2007-000278-22). Local research ethics review board approval has been obtained for each participating center.

The study is coordinated by the Epidemiology and Social Psychiatry Unit of the Mario Negri Institute for Pharmacological Research in collaboration with the Department of Mental Health of the Local Health Agency of Genoa and the Department of Psychiatry of the University of L'Aquila.

All study sites are monitored on a regular basis, case record forms are checked and data entered in the central database. According to Good Clinical Practice, all necessary procedures to ensure the quality of every aspect of the trial are complied with. Patient information is only accessible to the research team and no identifying information is kept with raw data. Each study investigator keeps records of laboratory tests and ECG diagrams in the patient's file as original source documents for the study.

All phases of the GiSAS trial will be recorded following the CONSORT statement [53].

\subsection{Study funding and data ownership}

The Italian Group for the Study of Second-generation Antipsychotics (GiSAS) was formed as a loose association in 2006 under the aegis of the Mario Negri Institute for Pharmacological Research, a non-profit research institute dedicated to health sciences research. The group currently comprises 21 members all of whom are part of the GiSAS trial steering board.

The GiSAS group started working on the protocol and the Mario Negri Institute accepted to act as sponsor for the study. As it was not possible obtaining economical support from national public agencies, funding was ensured through an unrestricted grant from the drug company Bristol-Myers Squibb. The grant was accepted by the Mario Negri Institute on the basis of a contract that guarantees full independence and data property. Bristol-Myers Squibb had no direct involvement in the study design, in the data collection, analysis and interpretation, in writing the present report or in the decision to submit it for publication. The GiSAS trial was acknowledged by all the ethics committees involved as an independent study aimed at improving clinical practice in health care, according to the definition of the Italian Ministerial Act 17 December 2004. This national law recognizes the public health value of not-for-profit studies on clinically relevant topics. If certain specific and accountable criteria of independence are met, the National Health Service supports the study conduct, covering part of the expenses, like the costs of study drugs and insurance. This permits a large multicenter trial on a relatively low budget, with no need to rely only on industry funding.

An independent review board has been set up to examine ethical issues related to the trial. All study data belong to the GiSAS trial investigators' group who undertake to publish them as soon as possible. Data will be analyzed and filed by the Epidemiology and Social Psychiatry Unit of Mario Negri Institute. Following publication of study results, full data will be made entirely available to the scientific community through unrestricted access to the trial database [54].

\subsection{Study progress}

At the date of revision of this manuscript 43 Italian centers were active in the study and 54 study investigators had included at least one patient. At the end of February 2011, the 260th patient was randomized. In total, 170 patients (65\%) had completed the one year follow-up, 10 (4\%) had dropped out and 80 (31\%) were still in active follow-up. All available baseline and follow-up data of the first 120 included patients had already been checked for accuracy and entered in the study database. The last subject should be included on March 2011 and the trial will be finished by mid-2012. Study data are entered as soon as they are collected, so the first results will be available by the end of 2012 . 


\section{Discussion}

The purpose of the study was to compare FGAs and SGAs in terms of tolerability and effectiveness with the goal of detecting clinically meaningful differences. Thus, the choice of study drugs fell on three antipsychotics with different backgrounds and completely different tolerability profiles, primarily aiming at identifying clinically meaningful differences.

Two recent Cochrane reviews on antipsychotic treatment for schizophrenia compared the effect of aripiprazole with SGAs or FGAs [55,56]. Aripiprazole was less effective than olanzapine, though only in terms of general mental state and only in the short term [55]. It also caused fewer side-effects like cholesterol increase, weight gain, sedation and prolactinrelated problems [55]. These findings are consistent with the meta-analysis already mentioned on metabolic side-effects of SGAs [13]. That review, in fact, found that aripiprazole was tolerated better than olanzapine in terms of weight gain and glucose and cholesterol elevation. Both analyses, however, were based on only two industry-sponsored RCTs and were biased by selective reporting $[49,57]$. Compared with FGAs, aripiprazole differed little in terms of efficacy but presented advantages in tolerability. It gave fewer EPS and less hyperprolactinemia and had an advantage in terms of attrition rates. Compared with haloperidol, however, it did not show any real advantage in terms of weight gain [56].

Even in the light of new evidence we can therefore conclude that the planned comparisons are well-balanced and should produce innovative and clinically meaningful results.

Adverse reactions like metabolic disturbances or EPS are extremely common with antipsychotic treatment. Given that three landmark studies - CATIE, CUtLASS and EUFEST - found no substantial differences in effectiveness, we planned the present trial to find out whether any one of the selected antipsychotics would be followed best and would cause less harm.

Since metabolic side-effects of SGAs (i.e. weight gain, dyslipidemia and impaired fasting glucose) were emerging issues and since aripiprazole showed an at least promising metabolic profile we opted for the choice of metabolic syndrome as primary endpoint for tolerability. Endpoints selected for clinical trials must strike a balance between their scientific validity and their practical and clinical importance. The ICH E9 guideline on biostatistics in clinical trials states that safety/tolerability may sometimes be the primary variable, and will always be an important consideration [58]. Recently Tyrer and Kendall (2008) wrote that serious adverse effects of antipsychotics should be adopted as important outcome measures [45]. The decision to focus on tolerability and to adopt a harmful effect as primary negative outcome, although uncommon, could therefore be valuable for improving the pharmacological treatment of schizophrenia.

According to the ICH E9 guideline, we indicated one primary variable - metabolic syndrome - as primary endpoint [58]. However, as the study design and conduction focus on drug retention, the GiSAS trial takes account of two primary endpoints: one for tolerability and one for effectiveness. Both were used to estimate the sample size because together they should provide the most clinically important and convincing evidence directly related to the primary objective of the trial [46].
A trial recruiting subjects with severe, long-lasting disorders has to deal with previous and current pharmacological treatments. Patients entering the GiSAS trial should have a condition appropriate for changing their antipsychotic treatment. This inclusion criterion is the vital starting point of the trial: patients should be included in the study only if the current medication is somehow unsatisfactory. As in CATIE we decided to allow random assignment to the medication taken prior to study entry. Given the possibility of not changing medication, staying on the same antipsychotic had to be a viable clinical option. On the other hand, those who were completely satisfied with their medication would not have entered the study. GiSAS participants who were already taking olanzapine, haloperidol or aripiprazole at baseline should therefore be not completely satisfied with their medication but did not need absolutely to change it. The use of the ITT principle allows to preserve randomization from any subsequent change of the allocated drug. Thus, if continuing previous medication will turn out to be the wrong choice clinicians will easily intervene without affecting trial participation.

The most important principle underlying RCTs is the concept of clinical equipoise which holds that randomization is appropriate when the clinician has substantial uncertainty as to which treatment is likely to provide greatest clinical benefit. We adopted this principle, which is the cornerstone of the credibility of all clinical trials, as the leading criterion for GiSAS recruitment. Thus, a patient was excluded if one of the study drugs had already proven ineffective or intolerable or if the treating clinician had any definite idea of which antipsychotic would be best.

Some characteristics of the study design might be sources of bias. Patients and their treating psychiatrists were unmasked for the assigned treatment, since this better reflects routine clinical practice increasing the trial's external validity. Knowledge of the treatment allocation and drug prescribed, however, can influence the referring clinician's and participant's assessment and interpretation of effectiveness and side-effects, and this might in turn influence treatment decisions and patients' subsequent use of the service and outcomes. The only strategy we could adopt to compensate this bias was to survey clinicians' attitudes towards antipsychotics with the aim of using these data to control for possible confounders. A secondary statistical analysis of the primary outcomes will adjust for clinicians' expectations of the effectiveness and tolerability of the various antipsychotic classes.

Post-randomization exclusion is another problematic issue related to the open nature of the trial. We decided to include in the ITT analysis only patients who had taken at least one dose of study medication. Thus, since patients and their psychiatrists were unblinded, they could choose to opt out of the trial if their treatment did not turn out to be the one they wanted. To deal with this, investigators were always prompted to adhere to the best possible standards of trial execution. All records on the randomization database (e.g. failed calls, unregistered and registered randomizations) are monitored by the study team on a weekly basis in order to detect any attempts to decipher allocation and to actively prompt clinicians to prescribe the allocated drug. At this stage, dropping out of the trial is not an easy option for the 
clinician and is considered acceptable only if patients withdraw informed consent. Moreover, patients for whom the allocated treatment turns out to be the same cannot be excluded from the ITT analysis since, by definition, they have already taken at least one dose of the investigational drug.

In CATIE phase I, $23 \%$ of subjects randomly assigned to olanzapine and $18 \%$ of subjects randomly assigned to risperidone did not change medication because they already were on those drugs at study entry. In a post-hoc study, Essock and colleagues (2006) found that those "stayers" had significantly longer times until discontinuation than those assigned to switch, and that, when these "stayers" were removed, differences seen in the original CATIE results were attenuated [59]. The authors concluded that future randomized comparisons should take into account whether medications being compared were newly initiated or not. Exposure to treatment prior to the trial seemed, in fact, to advantage "stayers", and this could represent another important source of bias which we will take into consideration.

\section{Conclusion}

For the last four years, the GiSAS study group has been committed to the implementation of this multicenter, pragmatic RCT on aripiprazole, olanzapine and haloperidol in the long-term treatment of schizophrenia. The trial mechanism is now fully functional and working, and the new recruitment target has been reached. Thus, we believe that trial will add useful knowledge on antipsychotic treatment for schizophrenic disorders.

\section{Competing interests}

The authors declare that they have no competing interests and that they have no past, current or pending financial link to any pharmaceutical company other than the research support administered by our Institution for the GiSAS trial conduct.

\section{Authors' contributions}

$\mathrm{AP}, \mathrm{BD}, \mathrm{MT}$ and $\mathrm{AB}$ contributed to the development of the study protocol. MT is the trial statistician and made an important contribution to the study design. AP wrote the first draft of the manuscript and, after revision, all authors approved the final manuscript. The content of the manuscript is solely the responsibility of the authors.

The Steering Committee approved the final draft of the study protocol, the Executive Committee supervised the trial's implementation and the Study Team managed the trial. The Study Investigators participated in enrolling patients.

\section{Acknowledgments}

The GiSAS trial is independently sponsored by the Mario Negri Institute for Pharmacological Research, which has received unrestricted funding from Bristol-Myers Squibb.

We thank Prof. Silvio Garattini, Director of the Mario Negri Institute, for the GiSAS trial promotion. We thank all members of the Steering Committee for their contribution to the preliminary protocol review process. In particular, we thank Prof. Clive Adams for his valuable help in the role of external advisor to GiSAS. We thank Dr. Luigi Ferrannini and Prof. Massimo Casacchia and all members of the GiSAS Study Team for their contribution in the trial coordination. We thank Ms. Judy Baggott for language editing.

\section{References}

[1] Adams CE, Awad G, Rathbone J, Thornley B. Chlorpromazine versus placebo for schizophrenia. Cochrane Database Syst Rev (2007), p. CD000284

[2] Joy CB, Adams CE, Lawrie SM. Haloperidol versus placebo for schizophrenia. Cochrane Database Syst Rev (2006), p. CD003082.

[3] Miyamoto S, Duncan GE, Marx CE, Lieberman JA. Treatments for schizophrenia: a critical review of pharmacology and mechanisms of action of antipsychotic drugs. Mol Psychiatry 2005;10:79-104.

[4] Leucht S, Pitschel-Walz G, Abraham D, Kissling W. Efficacy and extrapyramidal side-effects of the new antipsychotics olanzapine, quetiapine, risperidone, and sertindole compared to conventional antipsychotics and placebo. A meta-analysis of randomized controlled trials. Schizophr Res 1999;35:51-68.

[5] Geddes J, Freemantle N, Harrison P, Bebbington P. Atypical antipsychotics in the treatment of schizophrenia: systematic overview and meta-regression analysis. BMJ 2000;321:1371-6.

[6] Newcomer JW. Abnormalities of glucose metabolism associated with atypical antipsychotic drugs. J Clin Psychiatry 2004;65(Suppl 18): S36-46.

[7] Chakos M, Lieberman J, Hoffman E, Bradford D, Sheitman B. Effectiveness of second-generation antipsychotics in patients with treatmentresistant schizophrenia: a review and meta-analysis of randomized trials. Am J Psychiatry 2001;158:518-26.

[8] Tuunainen A, Wahlbeck K, Gilbody S. Newer atypical antipsychotic medication in comparison to clozapine: a systematic review of randomized trials. Schizophr Res 2002;56:1-10

[9] Adams CE, Jayaram M. Do findings from new trials for schizophrenia fit with existing evidence: not duped...just beguiled? Epidemiol Psichiatr Soc 2007;16:199-202.

[10] Duggan M. Do new prescription drugs pay for themselves? The case of second-generation antipsychotics. J Health Econ 2005;24:1-31.

[11] Montgomery JH, Byerly M, Carmody T, Li B, Miller DR, Varghese F, et al. An analysis of the effect of funding source in randomized clinical trials of second generation antipsychotics for the treatment of schizophrenia. Control Clin Trials 2004;25:598-612.

[12] Heres S, Davis J, Maino K, Jetzinger E, Kissling W, Leucht S. Why olanzapine beats risperidone, risperidone beats quetiapine, and quetiapine beats olanzapine: an exploratory analysis of head-to-head comparison studies of second-generation antipsychotics. Am J Psychiatry 2006;163:185-94.

[13] Leucht S, Corves C, Arbter D, Engel RR, Li C, Davis JM. Second-generation versus first generation antipsychotic drugs for schizophrenia: a metaanalysis. Lancet 2009;373:31-41.

[14] Rummel-Kluge C, Komossa K, Schwarz S, Hunger H, Schmid F, Lobos CA, et al. Head-to-head comparisons of metabolic side effects of second generation antipsychotics in the treatment of schizophrenia: a systematic review and meta-analysis. Schizophr Res 2010;123:225-33.

[15] Hotopf M, Churchill R, Lewis G. Pragmatic randomised controlled trials in psychiatry. Br J Psychiatry 1999;175:217-23.

[16] Thornley B, Adams C. Content and quality of 2000 controlled trials in schizophrenia over 50 years. BMJ 1998;317:1181-4.

[17] Lieberman JA, Stroup TS, McEvoy JP, Swartz MS, Rosenheck RA, Perkins $\mathrm{DO}$, et al. Effectiveness of antipsychotic drugs in patients with chronic schizophrenia. N Engl J Med 2005;353:1209-23.

[18] McEvoy JP, Lieberman JA, Stroup TS, Davis SM, Meltzer HY, Rosenheck RA, et al. Effectiveness of clozapine versus olanzapine, quetiapine, and risperidone in patients with chronic schizophrenia who did not respond to prior atypical antipsychotic treatment. Am J Psychiatry 2006;163: 600-10.

[19] Jones PB, Barnes TR, Davies L, Dunn G, Lloyd H, Hayhurst KP, et al. Randomized controlled trial of the effect on Quality of Life of second- vs first-generation antipsychotic drugs in schizophrenia: Cost Utility of the Latest Antipsychotic Drugs in Schizophrenia Study (CUtLASS 1). Arch Gen Psychiatry 2006;63:1079-87.

[20] Lewis SW, Barnes TR, Davies L, Murray RM, Dunn G, Hayhurst KP, et al. Randomized controlled trial of effect of prescription of clozapine versus other second-generation antipsychotic drugs in resistant schizophrenia. Schizophr Bull 2006;32:715-23.

[21] Kahn RS, Fleischhacker WW, Boter H, Davidson M, Vergouwe Y, Keet IP, et al. Effectiveness of antipsychotic drugs in first-episode schizophrenia 
and schizophreniform disorder: an open randomised clinical trial. Lancet 2008;371:1085-97.

[22] Keefe RS, Bilder RM, Davis SM, Harvey PD, Palmer BW, Gold JM, et al. Neurocognitive Working Group. Neurocognitive effects of antipsychotic medications in patients with chronic schizophrenia in the CATIE Trial. Arch Gen Psychiatry 2007;64:633-47.

[23] Davidson M, Galderisi S, Weiser M, Werbeloff N, Fleischhacker WW, Keefe RS, et al. Cognitive effects of antipsychotic drugs in first-episode schizophrenia and schizophreniform disorder: a randomized, openlabel clinical trial (EUFEST). Am J Psychiatry 2009;166:675-82.

[24] Leucht S, Kissling W, Davis JM. Second-generation antipsychotics for schizophrenia: can we resolve the conflict? Psychol Med 2009;39: 1591-602.

[25] Stahl SM. Dopamine system stabilizers, aripiprazole, and the next generation of antipsychotics, part 2: illustrating their mechanism of action. J Clin Psychiatry 2001;62:923-4.

[26] El-Sayeh HG, Morganti C. Aripiprazole for schizophrenia. Cochrane Database Syst Rev (2004), p. CD004578.

[27] Osservatorio nazionale sull'impiego dei medicinali. L'uso dei farmaci in Italia-Rapporto nazionale anno 2006. Roma: 2007. http://www.agenziafarmaco.it/it/content/rapporti-osmed-luso-dei-farmaci-italia; access verified 24/11/2010.

[28] Taylor DM, McAskill R. Atypical antipsychotics and weight gain: a systematic review. Acta Psychiatr Scand 2000;101:416-32.

[29] Irving CB, Adams CE, Lawrie S. Haloperidol versus placebo for schizophrenia. Cochrane Database Syst Rev (2006), p. CD003082.

[30] Oosthuizen P, Emsley R, Emsley R, Jadri Turner H, Keyter N. A randomized, controlled comparison of the efficacy and tolerability of low and high doses of haloperidol in the treatment of first-episode psychosis. Int J Neuropsychopharmacol 2004;7:125-31.

[31] Leucht C, Kitzmantel M, Kane J, Leucht S, Chua WLLC. Haloperidol versus chlorpromazine for schizophrenia. Cochrane Database Syst Rev (2008), p. CD004278.

[32] Expert Panel on Detection, Evaluation, and Treatment of High Blood Cholesterol in Adults. Executive Summary of The Third Report of The National Cholesterol Education Program (NCEP) Expert Panel on Detection, Evaluation, And Treatment of High Blood Cholesterol In Adults (Adult Treatment Panel III). JAMA 2001;285:2486-2497.

[33] Brown S, Inskip H, Barraclough B. Causes of the excess mortality of schizophrenia. Br J Psychiatry 2000;177:212-7.

[34] Joukamaa M, Heliövaara M, Knekt P, Aromaa A, Raitasalo R, Lehtinen V. Schizophrenia, neuroleptic medication and mortality. Br J Psychiatry 2006;188:122-7.

[35] De Hert M, Schreurs V, Vancampfort D, VAN Winkel R. Metabolic syndrome in people with schizophrenia: a review. World Psychiatry 2009;8:15-22.

[36] Enger C, Weatherby L, Reynolds RF, Glasser DB, Walker AM. Serious cardiovascular events and mortality among patients with schizophrenia. J Nerv Ment Dis 2004;192:19-27.

[37] Ray WA, Chung CP, Murray KT, Hall K, Stein CM. Atypical antipsychotic drugs and the risk of sudden cardiac death. N Engl J Med 2009;15: 225-35.

[38] Dunayevich E, Ascher-Svanum H, Zhao F, Jacobson JG, Phillips GA, Dellva MA, et al. Longer time to antipsychotic treatment discontinuation for any cause is associated with better functional outcomes for patients with schizophrenia, schizophreniform disorder, or schizoaffective disorder. J Clin Psychiatry 2007;68:1163-71.

[39] Gitlin M, Nuechterlein K, Subotnik KL, Ventura J, Mintz J, Fogelson DL, et al. Clinical outcome following neuroleptic discontinuation in patients with remitted recent-onset schizophrenia. Am J Psychiatry 2001;158: 1835-42.

[40] Ascher-Svanum H, Faries DE, Zhu B, Ernst FR, Swartz MS, Swanson JW. Medication adherence and long-term functional outcomes in the treatment of schizophrenia in usual care. J Clin Psychiatry 2006;67: 453-60.

[41] Lacro JP, Dunn LB, Dolder CR, Leckband SG, Jeste DV. Prevalence of and risk factors for medication nonadherence in patients with schizophrenia: a comprehensive review of recent literature. J Clin Psychiatry 2002;63:892-909.

[42] Liu-Seifert H, Adams DH, Kinon BJ. Discontinuation of treatment of schizophrenic patients is driven by poor symptom response: a pooled post-hoc analysis of four atypical antipsychotic drugs. BMC Med 2005;3:21.

[43] Kinon BJ, Liu-Seifert H, Adams DH, Citrome L. Differential rates of treatment discontinuation in clinical trials as a measure of treatment effectiveness for olanzapine and comparator atypical antipsychotics for schizophrenia. J Clin Psychopharmacol 2006;26:632-7.

[44] Sheehan DV, Lecrubier Y, Sheehan KH, Amorim P, Janavs J, Weiller E, Hergueta T, Baker R, Dunbar GC. The Mini-International Neuropsychiatric Interview (M.I.N.I.): the development and validation of a structured diagnostic psychiatric interview for DSM-IV and ICD-10. J Clin Psychiatry 1998;59(Suppl 20):S22-33.

[45] Tyrer P, Kendall T. The spurious advance of antipsychotic drug therapy. Lancet 2009;373:4-5.

[46] Neuhäuser M. How to deal with multiple endpoints in clinical trials. Fundam Clin Pharmacol 2006;20:515-23.

[47] Endicott J, Spitzer RL, Fleiss JL, Cohen J. The global assessment scale. A procedure for measuring overall severity of psychiatric disturbance. Arch Gen Psychiatry 1976;33:766-71.

[48] Day JC, Wood G, Dewey M, Bentall RP. A self-rating scale for measuring neuroleptic side-effects. Validation in a group of schizophrenic patients. Br J Psychiatry 1995;166:650-3.

[49] McQuade RD, Stock E, Marcus R, Jody D, Gharbia NA, Vanveggel S, Archibald D, Carson WH. A comparison of weight change during treatment with olanzapine or aripiprazole: results from a randomized, double-blind study. J Clin Psychiatry 2004;65(Suppl 18):S47-56.

[50] Zipursky RB, Gu H, Green AI, Perkins DO, Tohen MF, McEvoy JP, Strakowski SM, Sharma T, Kahn RS, Gur RE, Tollefson GD, Lieberman JA. Course and predictors of weight gain in people with first-episode psychosis treated with olanzapine or haloperidol. Br J Psychiatry 2005; 187:537-43.

[51] De Hert MA, van Winkel R, Van Eyck D, Hanssens L, Wampers M, Scheen A, Peuskens J. Prevalence of the metabolic syndrome in patients with schizophrenia treated with antipsychotic medication. Schizophr Res 2006;83:87-93.

[52] Haupt DW. Differential metabolic effects of antipsychotic treatments. Eur Neuropsychopharmacol 2006;16(Suppl 3):S149-55.

[53] Moher D, Schulz KF, Altman DG. The CONSORT statement: revised recommendations for improving the quality of reports of parallel-group randomized trials. Lancet 2001;357:1191-4.

[54] Steinbrook R, Kassirer JP. Free the data. BMJ 2010;341:811-2.

[55] Komossa K, Rummel-Kluge C, Schmid F, Hunger H, Schwarz S, El-Sayeh HGG, Kissling W, Leucht S. Aripiprazole versus other atypical antipsychotics for schizophrenia. Cochrane Database Syst Rev (2009), p. CD006569.

[56] Bhattacharjee J, El-Sayeh HGG. Aripiprazole versus typical antipsychotic drugs for schizophrenia. Cochrane Database Syst Rev (2008), p. CD006617.

[57] Kane JM, Osuntokun O, Kryzhanovskaya LA, Xu W, Stauffer VL, Watson $\mathrm{SB}$, et al. A 28-week, randomized, double-blind study of olanzapine versus aripiprazole in the treatment of schizophrenia. J Clin Psychiatry 2009;70:572-81.

[58] ICH E9 Expert Working Group. Statistical principles for clinical trials. ICH harmonized tripartite guideline. Stat Med 1999;18:1905-42.

[59] Essock SM, Covell NH, Davis SM, Stroup TS, Rosenheck RA, Lieberman JA. Effectiveness of switching antipsychotic medications. Am J Psychiatry 2006;163:2090-5. 